Research Paper

\section{Integrability-based Free-Form Mirror Design}

\author{
KazUaKi Kondo ${ }^{\dagger 1}$ Yasuhiro Mukaigawa ${ }^{\dagger 2}$ \\ and YASUSHI YAGI ${ }^{\dagger 2}$
}

A considerable issue in designing catadioptric imaging systems is what shape the component mirrors should be formed. In this paper, we propose a new algorithm for a catadioptric imaging system that satisfies the desired projection using a free-form mirror. A free-form mirror sapressed desired projection using a freersembly of gradients is a flexible surface representation that can form various shapes including non-smooth surfaces. We improve the shape reconstruction framework in the photometric stereo scheme to design free-form mirrors. An optimal mirror shape is formed to produce the desired projection under the integrability condition that requires it to be a consistent surface. We assume various catadioptric configurations, for which actual free-form mirrors are designed. The design experiments confirm that the resulting free-form mirrors can approximate the desired projections, including non-smooth ones.

\section{Introduction}

Using a curved mirror is one of the most powerful approaches for changing the projection of a primal imaging system such as a camera or projector. Researchers have proposed catadioptric imaging systems with specially formed mirrors to obtain particular projections used in various applications, including robot navigation and wide view surveillance. A typical use of the curved mirror is an omnidirectional imaging system. Conventional omnidirectional imaging systems use mirrors with basic convex shapes ${ }^{1)-3)}$, or special rotationally symmetric shapes to obtain the desired resolution in a vertical direction ${ }^{4)-6), 8}$. Gaechter, et al. used not only such a mirror but also a space variant imager to control image resolution ${ }^{12)}$. Kondo, et al. designed an asymmetric convex mirror to obtain an anisotropic projection property ${ }^{11)}$. The shapes of all the mirrors used in

$\dagger 1$ Kyoto University

$\nmid 2$ Osaka University the above proposals were defined by parameterized functions, resulting in limited shape representation and projection forming capabilities. A generalized representation of a mirror surface with a high DOF, together with a knowledge of its design, is necessary for producing arbitrary projections.

A free-form mirror determined from multiple control variables is one of the most generalized representations. It can be used to realize, without distortion, various projections given by practical configurations, such as a wide panoramic view. In this case, the desired projection is a uniform projection onto a cylindrical scene around the camera. A further example of the use of a free-form mirror is to correct the image distortion in projector systems. The mirror produces counterwarping against the distortion.

There may however, still be a problem with using a free-form mirror surface, in that there may not be a mirror that completely satisfies the desired projection. This was known as integrability of a gradient field on differential geometry. Hicks, et al. discussed the existence of a mirror surface that satisfies a given projection with Frobenius theorem, and used it to obtain an optimal existent mirror surface $^{7), 9), 10)}$. They defined a mirror surface as a polynomial function based on the Rayleigh-Ritz approximation method. Another algorithm for designing free-form mirrors was proposed by Swaminathan, et al. ${ }^{13)}$. They modeled the shape of the mirror using the cross products of splines to formulate the design problem as a linear equation. However, these conventional approaches had a problem representing non-smooth surfaces because of their smoothness property and the limited number control parameters that determined the design DOF.

In this paper, we propose a new surface definition for free-form mirrors, constructed as the assembly of its gradients. This type of free-form definition can represent mirror shapes, including non-smooth surfaces, more generally than the conventional algorithms, thus allowing a variety of projections to be produced. An example of this is a compound mirror constructed from multiple mirrors, such as a polygon mirror. This type of free-from mirror can produce a uniform projection onto a non-smooth scene such as a square room. Of course, compound mirrors constructed from curved surfaces can be defined as a combination of the free-form definition. Our method can design such a compound mirror directly as if it were a single mirror surface, with the result that we do not have to consider 
159 Integrability-based Free-Form Mirror Design

the joints between multiple mirrors. A spiny convex mirror is one of the nonsmooth surface mirrors. It produces an effective horizontal omnidirectional FOV for the omnidirectional camera.

A similar issue also occurs in the $3 \mathrm{D}$ shape reconstruction stage of photometric stereo schemes, in which the shape of a target object is estimated according to the shadings in an input image. On the other hand, the free-form mirror design algorithm estimates the optimal shape of a mirror to produce the desired projection. These two problem specifications are very similar. However, it is not feasible to apply the methods employed in the 3D shape reconstruction approach directly based on observed data into a mirror design scheme. The reason is that the meaning of the residuals is different in each problem. Additionally the assumption of an orthographic camera is also a problem in catadioptric imaging systems. For this reason, the algorithms used in the conventional shape reconstruction framework from its gradient field are extended for application in the mirror design problem. The results are tested experimentally.

\section{Problem Settings}

\subsection{Problem Specification for Mirror Design}

Mirror design for catadioptric imaging systems begins with defining the desired projection. Now assuming a camera as the primal imaging system, a capturing system is constructed. A projection system can be expressed as the reverse of a capturing system based on the theory that the light geometry is maintained even if the direction thereof is assumed to be reversed. Each incoming ray from a scene is reflected at some point on the free-form surface, and its captured position on the image plane is $(u, v)$. Then the desired projection can be expressed by rays in the scene $\mathbf{P}_{\mathbf{S}}(u, v)$ defined on the image plane, and can be freely manipulated by a designer. Similarly, projections of the primary camera are expressed as $\mathbf{P}_{\mathbf{c}}(u, v)$. Figure 1 illustrates this configuration. In this paper, we assume that the mirror size is small enough with respect to the environment. The desired projection is then indicated purely by the direction of the rays. $\mathbf{P}_{\mathbf{s}}(u, v)$ and $\mathbf{P}_{\mathbf{c}}(u, v)$ are related by reflection on a curved mirror placed in front of the camera. This is given by

$$
\mathbf{N}(u, v)=-\mathcal{N}\left[\mathcal{N}\left[\mathbf{P}_{\mathbf{s}}(u, v)\right]+\mathcal{N}\left[\mathbf{P}_{\mathbf{c}}(u, v)\right]\right]
$$

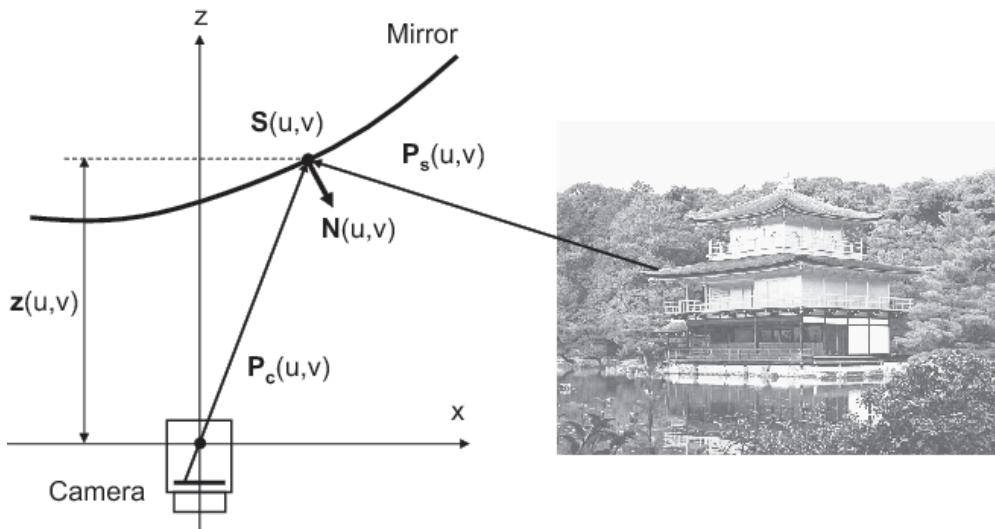

Fig. 1 Camera-Mirror configuration. A curved mirror $\mathbf{M}$ is placed on the front of a perspective projection camera $\mathbf{C}$. The mirror $\mathbf{M}$ should be formed so as to produce a normal vector field $\mathbf{N}(u, v)$ from a camera projection $\mathbf{P}_{\mathbf{c}}(u, v)$ and a given scene-to-image projection $\mathbf{P}_{\mathbf{s}}(u, v)$.

with the vector normalization operator $\mathcal{N}[\mathbf{x}]=\frac{\mathbf{x}}{\|\mathbf{x}\|}$, where $\mathbf{N}(u, v)$ is a normal vector field of the mirror surface. The aim in mirror design is to determine the surface that produces $\mathbf{N}(u, v)$. This is achieved by integrating gradient vectors from the normal vector field $\mathbf{N}(u, v)$.

Generally, gradients of a smooth surface can be determined by differentiation. However, the inverse, integrating the given gradients to reconstruct an original surface, is not always possible. Since an integration result depends on its path, integrations along different paths may (and in most cases do) produce divergent results when freely distributed gradients are given. To form a consistent surface, one needs to consider this problem.

\subsection{Problem Specification in the Shape Reconstruction Stage of a \\ Photometric Stereo Schem}

Photometric stereo schemes have a similar problem to mirror design, in that these schemes reconstruct a surface in two steps. First, a normal vector field of the target object is estimated from images under different illumination. Then the shape is estimated (reconstructed) from the obtained normal vectors. Strictly speaking, photometric stereo implies the former estimation based on shadings on 
the target object. But this stage is not important in this paper. Strongly related to the proposed approach is the latter stage in which shape reconstruction takes place after the estimation of a gradient field (or normal field).

Gradients are normally integrated to reconstruct the shape of the original object. Various methods have been proposed to assist in this process. These include solving a two-dimensional Poisson equation (this is the easiest and most popular), an iterative approach with the Gauss-Seidel method as per Horovitz, et al. ${ }^{14)}$, and a linear method from Agrawal ${ }^{15)}$.

\subsection{Similarities and Differences in the Problem Specifications}

As described in Sections 2.1 and 2.2, despite mirror design and shape reconstruction schemes are very different projects, interestingly, they result in very similar problems. We make use of this similarity in this paper. However, using the same approach proposed in conventional shape reconstruction schemes has disadvantages for mirror design.

The normal vector field, estimated from input images in a photometric stereo scheme, is generated from a real object. So, in principle, the shape of the object surface can be perfectly reconstructed if the images do not include any errors. If the integration cannot be performed consistently, this is due to image errors caused by, for instance, specular reflections, shadows, miscalibration, bad approximations, and so on. Decreasing the effect of image errors is, therefore, important in this type of shape reconstruction problem. Actually, the shape reconstruction method proposed by Agrawal ${ }^{15)}$ focused on eliminating errors such as impulse and inconsistent noise.

For mirror design, whether or not the normal vector field produced from the desired projection $\mathbf{P}_{\mathbf{s}}(u, v)$ can form a continuous consistent surface is a problem. Generally, the desired projection $\mathbf{P}_{\mathbf{s}}(u, v)$ is given without considering the possibility of forming a consistent surface. Therefore, in most cases, a solution that perfectly satisfies a given desired projection $\mathbf{P}_{\mathbf{s}}(u, v)$ does not exist, which is very different to the shape reconstruction problem that is based on observed data. The reason why integration of the gradients does not form a consistent surface is the given projection itself. In this case, an approach that minimizes the overall projection residual is recommended.

Furthermore, basic conventional shape reconstruction schemes assume known boundary conditions and an orthographic projection at the stage of normal vector estimation. In free-form mirror design, the designer has no information about the shape, or its boundary conditions. A perspective projection should be assumed in catadioptric imaging systems because an orthographic projection gives a bad approximation for mirrors placed close to the camera. The mirror design problem for catadioptric imaging systems requires a method under the perspective projection without any boundary conditions. Thus the conventional gradient based shape reconstruction approaches used in, for example, photometric stereo schemes, need to be extended for use in mirror design.

\section{Design of a Free-form Mirror}

\subsection{Representation of the Free-form Mirror}

In this paper we assume that a target catadioptric imaging system is constructed with a conventional perspective camera $C$ and a single reflective mirror $M$ placed in front of the camera (Fig. 1). The camera $C$ has a $W \times H$ size image plane $(u, v)$ that corresponds to a pixel on the image plane. The camera projection vector field $\mathbf{P}_{\mathbf{c}}(u, v)$ is given by

$$
\mathbf{P}_{\mathbf{c}}(u, v)=\left[\begin{array}{c}
-K_{u} u \\
-K_{v} v \\
f
\end{array}\right]
$$

where $f$ is the focal length of the camera and $K_{u}, K_{v}$ the size of the pixels. We assume a depth of field $z(u, v)$ defined on the image plane to form the shape of the mirror surface $\mathbf{S}(u, v)$ :

$$
\mathbf{S}(u, v)=\frac{1}{f} \mathbf{P}_{\mathbf{c}}(u, v) z(u, v) .
$$

We represent the free-form mirror shape with gradients $p=\frac{\partial z}{\partial u}, q=\frac{\partial z}{\partial v}$. The objective of the mirror design is to optimize $\mathbf{S}(u, v)$ by moving $p, q$ so as to produce $\mathbf{N}(u, v)$ with a minimum residual.

\subsection{Integrability of a Free-form Surface}

The reconstruction or design of a $3 \mathrm{D}$ surface must be done in such a way as to form a consistent surface. This is an implicit constraint that is referred to as the integrability of the surface ${ }^{16}$. On a consistent surface, integration of surface 
161 Integrability-based Free-Form Mirror Design

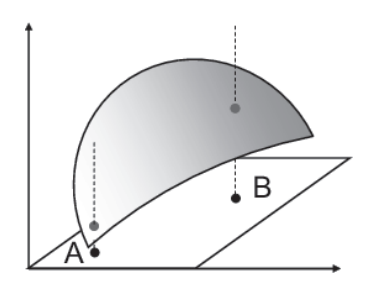

(a) two points on the surface

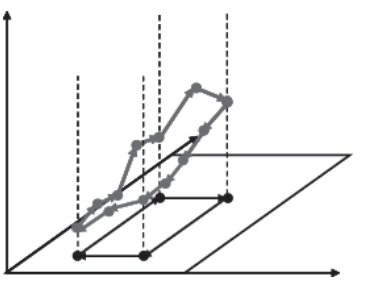

(c) integration along the closed loop

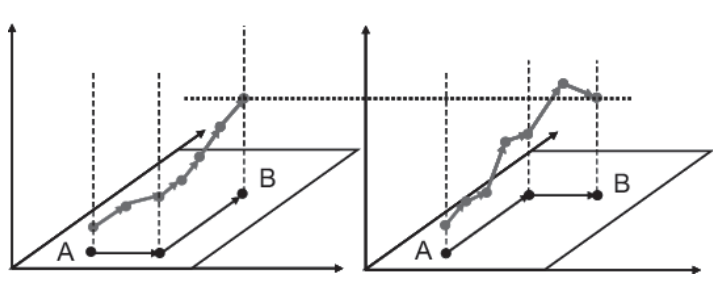

(b) integrations along different paths

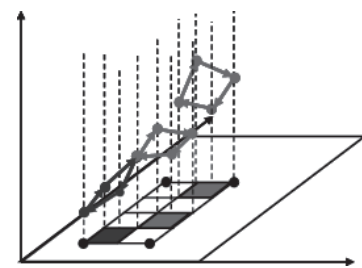

(d) linear combination of the unit loops
Fig. 2 A model of integrability on a surface. (b) Integration results from $A$ to $B$ along any path should be constant for a consistent surface. (c) It is equivalent to that integration results along any closed loop including $A$ and $B$ should be 0 . (d) Arbitrary closed loop can be expressed as a linear combination of the unit loops.

gradient vectors from a point $A$ to a point $B$ must be constant along any path as shown in Fig. 2. This is known as the integrability of a potential field ${ }^{16)}$. When an integration result along a path differs from that along any other path, the surface is discontinuous at that point.

Let $\mathbf{p}(u, v)$ and $\mathbf{q}(u, v)$ be gradients of $\mathbf{S}(u, v)$ in the $u$ and $v$ directions, $\frac{\partial S}{\partial u}$ and $\frac{\partial S}{\partial v}$, respectively. Integrability of the surface is generally formulated with a curl operator of $\mathbf{S}$ as

$$
\operatorname{curl}(\mathbf{p}, \mathbf{q})=\frac{\partial \mathbf{p}}{\partial v}-\frac{\partial \mathbf{q}}{\partial u}=\mathbf{0}
$$

at any point on $(u, v)$, thus representing the constraint for forming a consistent surface. This is well known as the integrability constraint. Agrawal, et al. also used the same formulation in their paper ${ }^{15)}$.

Remember that the shape of the mirror surface is given by Eq. (3). Since camera projection $\mathbf{P}_{\mathbf{c}}$ is determined by the camera parameters, and the only freedom in the shape $\mathbf{S}(u, v)$ is in the scalar field $z(u, v)$, an integrability constraint can be written in a scalar domain about $z$ instead of a vector domain $\mathbf{S}$. It is given by

$$
\operatorname{curl}(p, q)=\frac{\partial p}{\partial v}-\frac{\partial q}{\partial u}=0
$$

The mirror design must be performed with Eq. (5) to form a consistent surface. Here we define a target mirror surface using a set of continuous and sampled gradients on the $(u, v)$ field to formulate the design problem as linear equations. In this paper, the size of a pixel is assumed to denote the sampling interval, which states that a mirror surface is constructed by $2 W H$ gradients of $p$ and $q$ defined on a discrete domain $(u, v), u=0,1, \cdots, W-1, v=0,1, \cdots, H-1$. The following procedures are used to enforce the integrability constraint on the continuous domain over the discrete domain. Since the derivatives of the gradients are given as the differences of two contiguous values, the partial derivatives of $p$ and $q$ are obtained by

$$
\begin{aligned}
& \frac{\partial p}{\partial v}=p(u, v+1)-p(u, v) \\
& \frac{\partial q}{\partial u}=q(u+1, v)-q(u, v) .
\end{aligned}
$$

The equation for curl can be written in the discrete domain using Eq. (6). It is given by

$$
\text { curl }=p(u, v+1)-p(u, v)-q(u+1, v)+q(u, v) .
$$

One can write Eq. (7) in linear form as

$$
\text { curl }=[0, \cdots, 0,-1,0, \cdots, 0,1,0, \cdots, 0,1,-1,0, \cdots, 0] \mathbf{g}
$$

where $\mathbf{g}$ is a $2 W H \times 1$ vector constructed from the scan line ordered series of gradients $p$ and $q$ given as

$$
\begin{aligned}
& \mathbf{g}=[\quad p(0,0), p(1,0), \cdots, p(W-1,0), p(0,1), \cdots, p(W-1, H-1), \\
& q(0,0), q(1,0), \cdots, q(W-1,0), q(0,1), \cdots, q(W-1, H-1)]^{T}
\end{aligned}
$$

Equation (7) corresponds to a particular loop integral around a box of four pixels. Note that the loop integral around any other bigger loop can be expressed as linear combinations of the elementary loop integrals and thus does not provide any additional information. Thus stacking Eq. $(8)=0$ on the entire $(u, v)$ field, one obtains a linear equation for the integrability constraint:

$$
\mathrm{Cg}=\mathbf{0}
$$


162 Integrability-based Free-Form Mirror Design

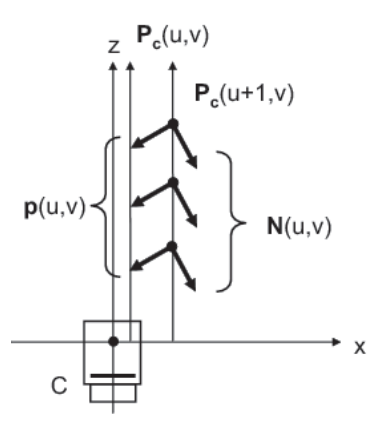

(a)

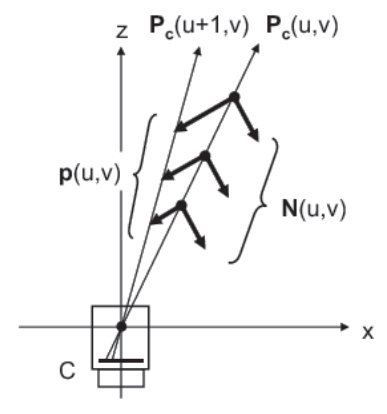

(b)
Fig. 3 Relationship between a normal vector and a gradient vector. (a) and (b) show models of orthographic projection and perspective projection cameras. The gradient vector in (a) is constant, independent of its depth. In (b) depth is ambiguous.

$\mathbf{C}$ is a sparse matrix of size $(W-1)(H-1) \times 2 W H$. Each row in $\mathbf{C}$ is in accordance with Eq. (8) and has four non-zero values; two +1 's corresponding to $p(u, v+1)$ and $q(u, v)$, respectively, and two -1 's corresponding to $p(u, v)$ and $q(u+1, v)$, respectively. This formulation procedure is similar to that in Ref. 15).

\subsection{Integrability on a Perspective Projection}

For gradient calculations from the normal vectors, conventional research such as classical photometric stereo schemes have assumed an orthographic projection camera because a camera can be placed sufficiently far from the target object to approximate the perspective projection to an orthographic one. If an orthographic projection is assumed, we can easily calculate the gradients $p, q$ from the normal vector field $\mathbf{N}$ since $x=K_{u} u$ and $y=K_{v} v$ (Fig. $\mathbf{3}(\mathrm{a})$ ). However, in catadioptric imaging systems, curved mirrors are placed relatively close to the camera for compactness. Thus the perspective projection cannot be approximated to an orthographic one. This means a method is needed to calculate the gradients.

Generally a normal vector $\mathbf{N}$ can be decomposed into two perpendicular gradient vectors whose outer product becomes $\mathbf{N}$ itself. For a perspective projection, we can obtain the directions of the gradient vectors, but their lengths are not uniquely determined because they depend on their depth as shown in Fig. 3 (b). Tankus' proposal ${ }^{17), 18)}$ provides a hint to solve this problem. Fortunately, the relationship between the length of the gradient vector and its depth under perspective projection is linear, resulting in

$$
\begin{aligned}
& \hat{p}=\frac{1}{z} \frac{\partial z}{\partial u} \\
& \hat{q}=\frac{1}{z} \frac{\partial z}{\partial v}
\end{aligned}
$$

rather than $p=\frac{\partial z}{\partial u}$ and $q=\frac{\partial z}{\partial v}$.

Having obtained the above, consideration should be given to how the integrability constraint with $\hat{p}, \hat{q}$ is formulated. Consider that $\operatorname{curl}(\hat{p}, \hat{q})$ can be expressed with $\operatorname{curl}(p, q)$ as

$$
\begin{aligned}
\operatorname{curl}(\hat{p}, \hat{q}) & =\frac{\partial \hat{p}}{\partial v}-\frac{\partial \hat{q}}{\partial u} \\
& =\frac{\partial}{\partial v}\left(\frac{1}{z} \frac{\partial z}{\partial u}\right)-\frac{\partial}{\partial u}\left(\frac{1}{z} \frac{\partial z}{\partial v}\right) \\
& =-\frac{1}{z^{2}}\left(\frac{\partial z}{\partial v} \frac{\partial z}{\partial u}-\frac{\partial z}{\partial v} \frac{\partial z}{\partial u}\right)+\frac{1}{z}\left(\frac{\partial^{2} z}{\partial v \partial u}-\frac{\partial^{2} z}{\partial u \partial v}\right) \\
& =\frac{1}{z} \operatorname{curl}(p, q)
\end{aligned}
$$

Since $z>0, \operatorname{curl}(p, q)=0$ is equivalent to $\operatorname{curl}(\hat{p}, \hat{q})=0$. We can now say that curls in the discrete domain have the same characteristics as curls in the continuous domain. Thus a set of $\hat{p}$ and $\hat{q}$ can be used as $\mathbf{g}$ instead of $p$ and $q$ for the integrability constraint. The shape of the surface can easily be produced by integrating $\hat{p}$ and $\hat{q}$. Integration of $\hat{p}$ and $\hat{q}$ provides a log scaled shape, because

$$
\begin{aligned}
& \hat{p}=\frac{1}{z} \frac{\partial z}{\partial u}=\frac{\partial \ln z}{\partial u} \\
& \hat{q}=\frac{1}{z} \frac{\partial z}{\partial v}=\frac{\partial \ln z}{\partial v} .
\end{aligned}
$$

Note that the use of $\hat{p}$ and $\hat{q}$ requires an additional procedure when forming a shape from optimized gradients, namely the exponents of the integration results to convert a log scaled shape into an original shape. See Section 3.6 for more detail. In this paper, we use $\hat{p}$ and $\hat{q}$ as gradients with an assumption of a perspective projection.

Of course, we can attach a telecentric lens unit to the primal imaging system 
so as to generate the orthogonal projection. In that case, $p$ and $q$ can be used directly without considering $\hat{p}$ and $\hat{q}$.

\subsection{Gradient Optimization under the Integrability Constraint}

An optimal shape for the mirror surface should minimize residuals from the desired gradients under the integrability constraint from the previous section. The approach proposed by Agrawal ${ }^{15)}$ for the photometric stereo method uses a graph that describes the reliability of the gradients. Gradients with high reliability are retained and the others are ignored to satisfy the integrability constraint, much like an elimination of outliers. This proposal works well for the photometric stereo problem because the gradients obtained from the images can have unexpected errors. Conversely, residuals in the mirror design problem are not caused by unexpected errors but by non-integrability of the given desired gradients. Thus minimization of residuals all over is superior to the elimination of outliers.

Let $\mathbf{g}$ be a vector consisting of gradients $\hat{p}, \hat{q}$ that form a surface, and let $\mathbf{g}_{\mathbf{d}}$ be that of desired gradients $\hat{p_{d}}$ and $\hat{q_{d}}$ obtained from the desired projection. The goal for the optimization is to minimize the cost function $F\left(\mathbf{g}, \mathbf{g}_{\mathbf{d}}\right) \in \mathbf{R}^{\mathbf{1}}$ formulated as

$$
F\left(\mathbf{g}, \mathbf{g}_{\mathbf{d}}\right)=\left\|\mathbf{g}-\mathbf{g}_{\mathbf{d}}\right\|^{2}
$$

about $\mathbf{g}$ under the integrability constraint Eq. (10). In fact, gradient $\mathbf{g}$ does not have the same DOF as the number of its row, because of the constraint from Eq. (10). Therefore, g can be expressed as a general solution of Eq. (10) We formulate this with the following scheme. Assume a homogeneous linear equation $\mathbf{A x}=\mathbf{0}$ with an $m \times n$ matrix $\mathbf{A}(m<n$ and $\operatorname{rank} \mathbf{A}=m)$. This can be transformed into

$$
\left[\begin{array}{ll}
-\mathbf{E}_{\mathbf{m}} & \mathbf{B}
\end{array}\right] \mathbf{x}=\mathbf{0}
$$

with a basic matrix transformation of the rows of $\mathbf{A}$. Here $\mathbf{E}_{\mathbf{i}}$ is an $i \times i$ unit matrix. Then the general form of its solution is described as

$$
\mathbf{x}=\left[\begin{array}{c}
\mathbf{B} \\
\mathbf{E}_{\mathbf{n}-\mathbf{m}}
\end{array}\right] \mathbf{y}
$$

where $\mathbf{y}$ is a vector that denotes the actual DOF of the solution $\mathbf{x}$ for $\mathbf{A x}=\mathbf{0}$ g can be expressed as the same formulation as Eq. (16), in the case of Eq. (10) instead of $\mathbf{A x}=\mathbf{0}$. See the appendix for a detailed explanation of the above theory. Let the integrability constraint $\mathbf{C}$ and the gradient $\mathbf{g}$ correspond to $\mathbf{A}$ and $\mathbf{x}$, respectively, so that the above proposition can be used. Based on Eq. (8) and Eq. (10), C is formed as

$$
\mathbf{C}=\left[\begin{array}{ccccc|ccccc}
-\mathbf{P} & \mathbf{P} & & & \mathbf{0} & \mathbf{Q} & & & \mathbf{0} & \mathbf{0} \\
& -\mathbf{P} & \mathbf{P} & & & & \mathbf{Q} & & & \vdots \\
& & \ddots & \ddots & & & & \ddots & & \vdots \\
\mathbf{0} & & & -\mathbf{P} & \mathbf{P} & \mathbf{0} & & & \mathbf{Q} & \mathbf{0}
\end{array}\right]
$$

where $\mathbf{P}, \mathbf{Q}$, and $\mathbf{0}$ are $(W-1) \times W$ matrices as given below.

$$
\mathbf{P}=\left[\begin{array}{r}
0 \\
\mathbf{E}_{\mathbf{W}-\mathbf{1}} \\
\vdots \\
0
\end{array}\right], \mathbf{Q}=\left[\begin{array}{ccccc}
1 & -1 & & & 0 \\
& 1 & -1 & & \\
& & \ddots & \ddots & \\
0 & & & 1 & -1
\end{array}\right] \text {, and } \mathbf{0}=\left[\begin{array}{ccc}
0 & \cdots & 0 \\
\vdots & \ddots & \vdots \\
0 & \cdots & 0
\end{array}\right] \text {. }
$$

$\mathbf{C}$ is constructed from $(H-1) \times 2 H$ blocks of $\mathbf{P}, \mathbf{Q}$ and $\mathbf{0}$ as shown in Eq. (17). The construction of $\mathbf{C}$ indicates $\operatorname{rank} \mathbf{C}=(W-1)(H-1)$. Now assume another gradient vector $\overline{\mathbf{g}}$ with a different order for $\hat{p}$ and $\hat{q}$ to replace $\mathbf{P}$ in $\mathbf{E}_{\mathbf{W - 1}}$ and apply the theory of Eq. (15) and Eq. (16). This enables us to obtain a new form of the integrability constraint $\overline{\mathbf{C}} \overline{\mathbf{g}}=\mathbf{0}$, its ideal solution $\overline{\mathbf{g}_{\mathbf{d}}}$, and a cost function $\bar{F}$ with

$$
\begin{aligned}
\overline{\mathbf{g}}=[ & \hat{p}(0,0), \hat{p}(1,0), \cdots, \hat{p}(W-2,0), \\
& \hat{p}(0,1), \hat{p}(1,1), \cdots, \hat{p}(W-2,1), \\
& \hat{p}(0,2), \cdots, \hat{p}(W-2, H-1), \\
& \hat{p}(W-1,0), \hat{p}(W-1,1), \cdots, \hat{p}(W-1, H-1), \\
& \hat{q}(0,0), \hat{q}(1,0), \cdots, \hat{q}(W-1,0), \\
& \hat{q}(0,1), \hat{q}(1,1), \cdots, \hat{q}(W-1,1), \\
& \hat{q}(0,2), \cdots, \hat{q}(W-1, H-1)]^{T},
\end{aligned}
$$




$$
\begin{array}{cl}
\overline{\mathbf{g}} & \hat{p_{d}}(0,0), \hat{p_{d}}(1,0), \cdots, \hat{p_{d}}(W-2,0), \\
& \hat{p_{d}}(0,1), \hat{p_{d}}(1,1), \cdots, \hat{p_{d}}(W-2,1), \\
& \hat{p_{d}}(0,2), \cdots, \hat{p_{d}}(W-2, H-1), \\
& \hat{p_{d}}(W-1,0), \hat{p_{d}}(W-1,1), \cdots, \hat{p_{d}}(W-1, H-1), \\
& \hat{q_{d}}(0,0), \hat{q_{d}}(1,0), \cdots, \hat{q_{d}}(W-1,0), \\
& \hat{q_{d}}(0,1), \hat{q_{d}}(1,1), \cdots, \hat{q_{d}}(W-1,1), \\
& \left.\hat{q_{d}}(0,2), \cdots, \hat{q_{d}}(W-1, H-1)\right]^{T}, \\
\bar{F}\left(\overline{\mathbf{g}}, \overline{\mathbf{g}}(\overline{\mathbf{d}})=\|\overline{\mathbf{g}}-\overline{\mathbf{g}}\|^{2},\right.
\end{array}
$$

and

$$
\overline{\mathbf{C}}=\left[\begin{array}{ccccc|ccccc}
-\mathbf{E}_{\mathrm{W}-1} & \mathbf{E}_{\mathrm{W}-1} & & & 0 & \mathrm{Q} & & & 0 & 0 \\
& -\mathbf{E}_{\mathrm{W}-1} & \mathbf{E}_{\mathrm{W}-1} & & & & & & \\
& & \ddots & \ddots & & & & \\
& & & -\mathbf{E}_{\mathrm{W}-1} & \mathbf{E}_{\mathrm{W}-1} & 0 & & \ddots & & \vdots \\
0 & & & & 0
\end{array}\right]
$$

Take note of the left part of $\overline{\mathbf{C}}$. Working from the bottom to the top of the matrix, and sequentially adding a row block to the row block immediately above it, converts the left part into a negative unit matrix given by

$$
\begin{aligned}
& {\left[\begin{array}{ccccc|ccccc}
-\mathbf{E}_{\mathrm{W}-1} & & & \mathbf{0} & \mathbf{E}_{\mathrm{W}-1} & \mathbf{Q} & \mathbf{Q} & \cdots & \mathbf{Q} & 0 \\
& -\mathbf{E}_{\mathrm{W}-1} & & & \vdots & & \mathbf{Q} & \cdots & \mathbf{Q} & \vdots \\
& & \ddots & & \vdots & & & \ddots & \vdots & \vdots \\
0 & & & -\mathbf{E}_{\mathrm{W}-1} & \mathbf{E}_{\mathrm{W}-1} & \mathbf{0} & & & \mathbf{Q} & 0
\end{array}\right] \overline{\mathbf{g}}=\mathbf{0}} \\
& \Leftrightarrow\left[\begin{array}{ll}
-\mathbf{E}_{(\mathbf{W}-\mathbf{1})(\mathbf{H}-\mathbf{1})} & \mathbf{S}
\end{array}\right] \overline{\mathbf{g}}=\mathbf{0}
\end{aligned}
$$

where

$$
\mathbf{S}=\left[\begin{array}{cccccc}
\mathbf{E}_{\mathrm{W}-1} & \mathbf{Q} & \mathbf{Q} & \cdots & \mathbf{Q} & 0 \\
\vdots & & \mathbf{Q} & \cdots & \mathbf{Q} & \vdots \\
\vdots & & & \ddots & \vdots & \vdots \\
\mathbf{E}_{\mathrm{W}-1} & 0 & & & \mathbf{Q} & 0
\end{array}\right]
$$

corresponding to B in Eq. (15) and Eq. (16). Then the general solution defined by the integrability constraint is represented as

$$
\overline{\mathbf{g}}=\left[\begin{array}{c}
\mathrm{S} \\
\mathbf{E}_{\mathbf{2} \mathbf{W H}-(\mathrm{W}-\mathbf{1})(\mathbf{H}-\mathbf{1})}
\end{array}\right] \mathbf{h}=\mathbf{T h}
$$

based on Eq. (16). The $2 W H-(W-1)(H-1) \times 1$ vector $\mathbf{h}$ corresponds to $\mathbf{y}$ in Eq. (16). T is a $2 W H \times(2 W H-(W-1)(H-1))$ matrix whose columns are linearly independent solutions of Eq. (10). Substituting Eq. (25) into Eq. (21) for a new cost function $\overline{\mathbf{F}}\left(\mathbf{h}, \overline{\mathbf{g}_{\mathbf{d}}}\right)$ given as

$$
\bar{F}\left(\mathbf{h}, \overline{\mathbf{g}_{\mathbf{d}}}\right)=\left\|\mathbf{T h}-\overline{\mathbf{g}_{\mathbf{d}}}\right\|^{2}
$$

with actual DOF $\mathbf{h}$ instead of $\overline{\mathbf{g}}$. Here we use a simple Least Mean Square algorithm to minimize Eq. (25) and obtain the optimal solution for $\overline{\mathrm{g}}$. This is expressed as

$$
\mathbf{h}=\mathbf{T}^{+} \overline{\mathbf{g}_{\mathbf{d}}} \Rightarrow \overline{\mathbf{g}}=\mathbf{T T}^{+} \overline{\mathbf{g}_{\mathrm{d}}}
$$

where $\mathbf{T}^{+}$is the pseudo inverse matrix of $\mathbf{T}$. Since $\mathbf{T}^{+} \neq \mathbf{T}^{T}$, we must calculate $\mathbf{T}^{+}=\left(\mathbf{T}^{T} \mathbf{T}\right)^{-1} \mathbf{T}^{T}$. This procedure does not require any additional prior information despite the fact that typical gradient based 3D-shape reconstruction approaches, such as using a two dimensional Poisson equation and the algebraic method $^{15)}$, need the boundary conditions.

\subsection{Adaptive Optimization with a Weight Map}

By using a weight map that gives evaluation weights to each gradient, optimization becomes more adaptive. This is useful when a designer wishes to give a non-uniform importance map, or when the influence of gradient residuals on projection residuals changes according to location on the mirror surface ${ }^{13}$. A weight map that gives small weightings at locations with large residuals can suppress the expansion thereof. This countermeasure works effectively when a desired projection is discontinuous and/or bumpy.

A weight map can be generated by checking curl values of the gradient map obtained from the given desired projection in advance. The reason is that projection residuals tend to appear at locations with non-integrability gradients. Thus a small weight or even 0 should be given to these when generating the weight map.

Here we discuss differences between the proposed method and Agrawal's method ${ }^{15)}$ by considering the weight map. Since the integrability constraint partially prevents Eq. $(21)=0,2 W H-(W-1)(H-1)$ DOF, corresponding to $\mathbf{h}$ still remain. The proposed method uses the remaining DOF to obtain an optimal $\overline{\mathbf{g}}$ that minimizes square residuals from $\overline{\mathbf{g}_{\mathbf{d}}}$ all over the field $(u, v)$. On the other hand, Agrawal, et al. chained contiguous nodes to create edges in the graph show- 
ing the topology of the integrability constraint, so as to add $2 W H-(W-1)(H-1)$ requirements. This procedure is similar to allocating weights of 1 and 0 to the corresponding $2 W H-(W-1)(H-1)$ gradients and the other $(W-1)(H-1)$ gradients, respectively. As a result, the $2 W H-(W-1)(H-1)$ elements in $\overline{\mathbf{g}}$ become equal to those in $\overline{\mathbf{g}_{\mathbf{d}}}$, and the other $(W-1)(H-1)$ elements are automatically determined based on the integrability constraint without any effect from the corresponding elements in $\overline{\mathbf{g}_{\mathbf{d}}}$. Since this method has an all-or-nothing approach at each $(u, v)$, it cannot be used for overall optimization.

The weight map is defined as a $2 W H \times 2 W H$ matrix $\mathbf{M}$, the diagonal components of which are the weights for each gradient. Re-alignment of $\mathbf{g}$ to $\overline{\mathbf{g}}$ creates $\mathbf{M}$, with the same order, as

$$
\begin{aligned}
\mathbf{M}=\operatorname{diag}( & m_{p}(0,0), m_{p}(1,0), \cdots, m_{p}(W-2,0) \\
& m_{p}(0,1), m_{p}(1,1), \cdots, m_{p}(W-2,1) \\
& m_{p}(0,2), \cdots, m_{p}(W-2, H-1) \\
& m_{p}(W-1,0), m_{p}(W-1,1), \cdots, m_{p}(W-1, H-1) \\
& m_{q}(0,0), m_{q}(1,0), \cdots, m_{q}(W-1,0) \\
& m_{q}(0,1), m_{q}(1,1), \cdots, m_{q}(W-1,1) \\
& \left.m_{q}(0,2), \cdots, m_{q}(W-1, H-1)\right)
\end{aligned}
$$

where $m_{p}$ and $m_{q}$ are weights corresponding to gradients $\hat{p}$ and $\hat{q}$, respectively. In this paper, we took $m_{p}(u, v)$ and $m_{q}(u, v)$ to be the same value, $w(u, v)$, calculated as

$$
w(u, v)=1+\left(w_{\max }-1\right) \times \frac{\operatorname{Curl}_{\text {ave }}(u, v)}{\operatorname{Curl}_{\max }}
$$

in order to configure $1 \leq w(u, v) \leq w_{\max }$. Here $w_{\max }$ is the maximum weight value given by the designer. $\operatorname{Curl}_{\max }$ and $\operatorname{Curl}_{\text {ave }}(u, v)$ denote a maximum curl value on an entire $(u, v)$ field and an average of neighbor curl values around a target $(u, v)$ expressed as

$$
C u r l_{\max }=\max _{u, v} \operatorname{curl}(u, v)
$$

and

$$
\operatorname{Curl}_{\text {ave }}(u, v)=\frac{1}{4} \sum_{i=0}^{1} \sum_{j=0}^{1} \operatorname{curl}(u+j, v+i)
$$

respectively. Applying $\mathbf{M}$ to Eq. (26) achieves a weighted optimization.

$\bar{F}\left(\mathbf{h}, \overline{\mathbf{g}_{\mathbf{d}}}\right)=\left\|\mathbf{M T h}-\mathbf{M} \overline{\mathbf{g}_{\mathbf{d}}}\right\|^{2}$

$\mathbf{h}=(\mathbf{M T})^{+} \mathbf{M g}_{\bar{d}} \Rightarrow \overline{\mathbf{g}}=\mathbf{T}(\mathbf{M T})^{+} \mathbf{M g}_{\bar{d}}^{-}$

Although $\mathbf{M}^{-1}=\operatorname{diag}\left(\frac{1}{m_{p}(0,0)}, \cdots, \frac{1}{m_{q}(W-1, H-1)}\right),(M T)^{+}$cannot be obtained by any multiplications thereof, such as $\mathbf{M}^{-1}, \mathbf{T}^{T}, \mathbf{T}^{+}$, and so on. Therefore we need to calculate $(\mathbf{M T})^{+}=\left((\mathbf{M T})^{T}(\mathbf{M T})\right)^{-1}(\mathbf{M T})^{T}$.

This adaptive optimization using the weight map can be applied to conventional approaches ${ }^{10), 13)}$. However, the correspondences between the control variables and the gradients on the mirror surface are not one-to-one. Thus the weight map does not contribute appropriately to forming the mirror in these approaches. Since each weight has a direct affect on every location in our method, the gradient optimization is done more adaptively.

\subsection{Forming the Mirror Shape from Its Gradients}

Forming the mirror shape takes place after the optimal gradients have been obtained. Since the relative positions of contiguous points on the surface are determined by the gradients, giving an integral constant value allows us to form the mirror shape. This is given as the depth of a point $z_{0}=z\left(u_{0}, v_{0}\right) \quad 0<u_{0}<$ $W-1,0<v_{0}<H-1$. Depths at the other points $(u, v)$ can be calculated by sequential additions (integrations) of the optimized gradients along a path from $\left(u_{0}, v_{0}\right)$ to $(u, v)$. Note that the path of the integration does not affect the integration result, because the optimized gradients satisfy the integrability constraint.

Define $p_{o}$ and $q_{o}$ as optimized gradients, in the case of an orthogonal projection camera, to formulate the integration procedure given by

$$
z\left(u, v_{0}\right)=\left\{\begin{array}{cc}
z_{0}+\sum_{j=u_{0}}^{u-1} p_{o}\left(j, v_{0}\right) & u>u_{0} \\
z_{0}-\sum_{j=u}^{u_{0}-1} p_{o}\left(j, v_{0}\right) & u<u_{0}
\end{array}\right.
$$

and 
166 Integrability-based Free-Form Mirror Design

$$
z(u, v)=\left\{\begin{array}{cc}
z\left(u, v_{0}\right)+\sum_{i=v_{0}}^{v-1} q_{o}(u, i) & v>v_{0} \\
z\left(u, v_{0}\right)-\sum_{i=u}^{v_{0}-1} q_{o}(u, i) & v<v_{0}
\end{array}\right.
$$

When a perspective projection is assumed to be a camera projection, the optimal gradients are said to be $\log$ scaled. This indicates that $\hat{z_{0}}=\ln z_{0}, \hat{z}=\ln z, \hat{p_{o}}$, and $\hat{q}_{o}$ are used instead of $z_{0}, z, p_{o}$, and $q_{o}$ in the above integration, respectively. Additional exponent calculation is necessary to convert the log scaled depth $\hat{z}(u, v)$ into an original depth $z(u, v)$. Since the designer can arbitrarily give a depth and its position, the distance from the mirror and the camera can be controlled.

\section{Design Experiments}

\subsection{Wide Panoramic Imaging System}

The free-form mirrors designed from our algorithm satisfy both an expanding FOV and reduced distortion on captured images. We assumed a configuration for a wide panoramic imaging system as shown in Fig. 4(a). In this setup, distortion-free means that cylindrical scenes around the camera appear directly on the images. A desired projection is given by dividing an objective panoramic FOV into grids of the same size. The FOV of the camera and the objective panoramic FOV are respectively, $\pm 20.0 \times \pm 15.3$ degrees produced with a $6.0 \mathrm{~mm}$ focal length, and $\pm 50.0 \times \pm 20.0$ degrees. The shape of the designed mirror, a target panoramic scene, and a captured image are shown in Fig. 4. Checkers on the cylindrical scene appear approximately as squares on the image. The center region has almost no distortion, whereas a little distortion appears at the image edges. The distortion is, however, much smaller than that which results from conventional parametric approaches such as using rotationally symmetric mirrors.

\subsection{Catadioptric Projector System}

Free-form mirrors can also correct image distortions in projector systems. Generally direct projection by an upward facing projector has trapezoidal distortion. Using a combination of a projector and a free-form mirror, rectangular images appear on a screen without image deformation, and these cancel the trapezoidal distortion. Since the projection of a projector is the same as that of a camera

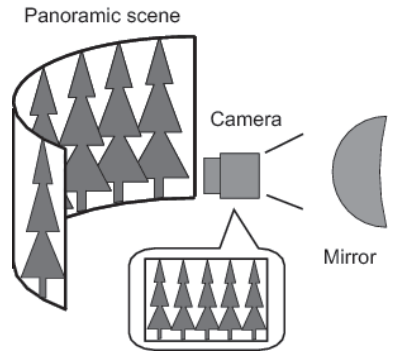

(a) setup

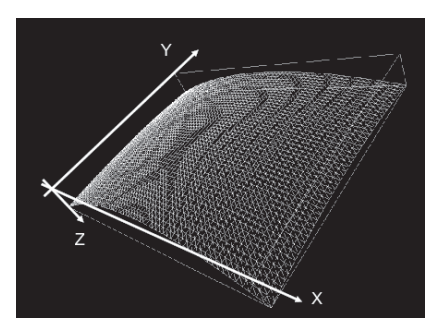

(b) designed mirror

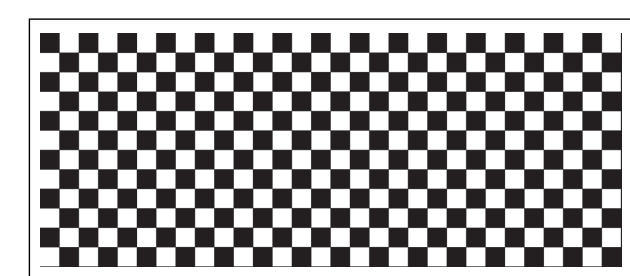

(c) target panoramic scene

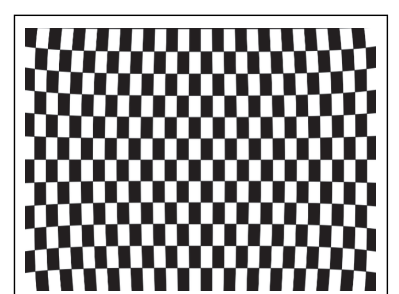

(d) input image
Fig. 4 Wide panoramic imaging system. $X, Y$, and $Z$ axes in figure (b) correspond to the camera coordinate system.

apart from the direction of the light, our algorithm can be applied directly. Assume a catadioptric projector system as shown in Fig. 5 (a). We configured a rectangular projection as the desired one, and designed the catadioptric projector system using our algorithm. The focal length and FOV of the projector are the same as those for the camera in the previous experiment. The shape of the designed mirror and examples of projected images are shown in Fig. 5 (b) and Fig. $5(\mathrm{c}-\mathrm{e})$, respectively. The trapezoidal distorted image with only the upward projector is corrected with the use of the designed free-form mirror to be a rectangular image. Although a little distortion still remains, the amount of image deformation required to cancel it is much smaller.

\subsection{Anisotropic Omnidirectional Camera}

The previous two configurations aim to achieve uniform and orthogonal projections. Here we demonstrate an optical construction that has a more particular projection. Generally, most catadioptric omnidirectional cameras use rotational 


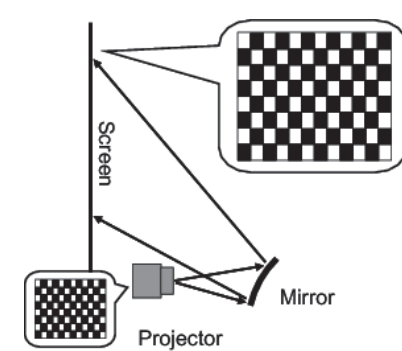

(a) setup

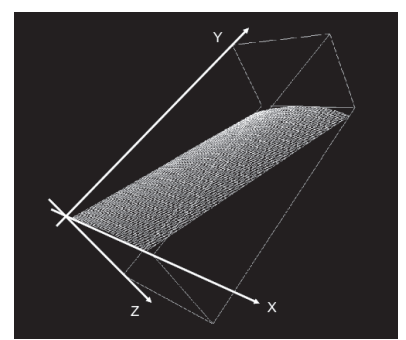

(b) designed mirror

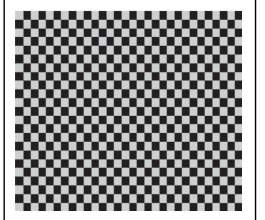

(c) ideal project

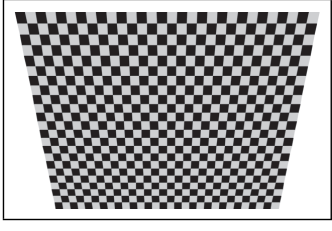

(d) only a looking up projector

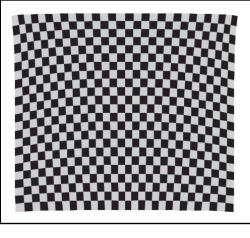

(e) with the designed mirror
Fig. 5 Catadioptric projector system.

symmetric convex mirrors and align these to the optical axis of the camera. Thus conventional omnidirectional cameras have isotropic optical and geometrical properties.

There are some cases in which anisotropic properties work well. One example is vision in mobile robots, where it is desirable for the robot to observe the direction along its moving path to avoid collisions, recognize targets, manipulate objects, and so on. An anisotropic omnidirectional imaging system with continuously changing resolution along the azimuth angle to observe the scenes in the front and back with higher resolution than those on the sides has been proposed by Kondo, et al. ${ }^{11)}$. We have also designed an anisotropic omnidirectional camera with a convex mirror designed from our algorithm. Input images of a conventional isotropic omnidirectional camera and our anisotropic one are shown in Fig. 6 (d) and (e), respectively, with the target being a uniform checker pattern on the panoramic scene. While the same size checkers appear concentrically in Fig. 6(d), larger checkers appear on the left and right of Fig. 6 (e) than at the

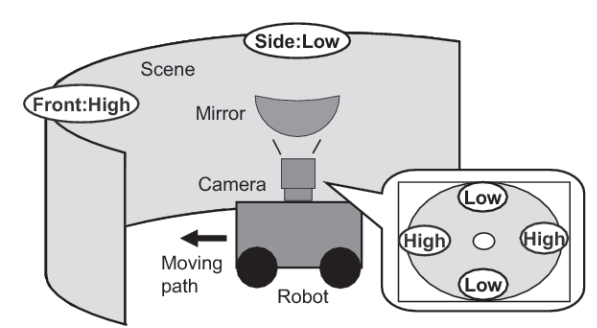

(a) objective anisotropic projection

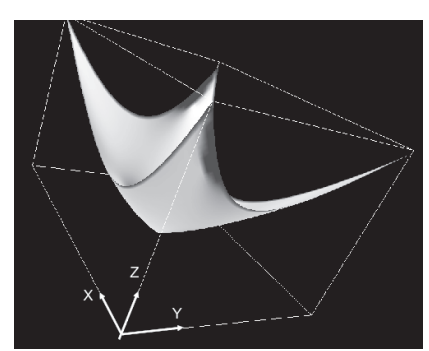

(b) designed mirror

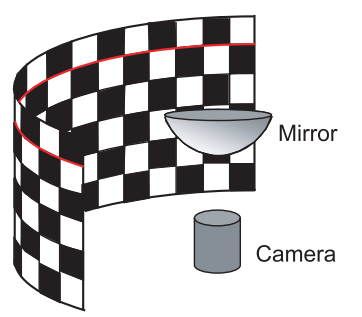

(c) setup for generating input images

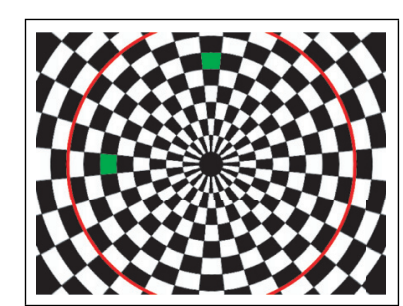

(d) input image with the isotropic mirror

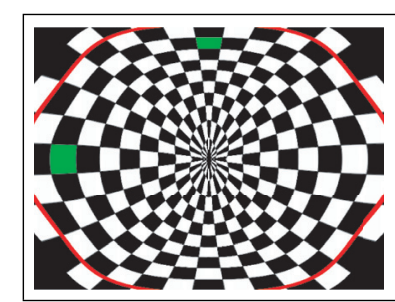

(e) input image with the designed mirror
Fig. 6 Anisotropic omnidirectional camera for mobile robots. The radial and concentric direction on the input images (d), (e) correspond to the latitudinal and longitudinal direction in the world, respectively. Red lines correspond to horizontal rings in the world. Typical checkers located in a perpendicular direction are colored green for comparison.

top and bottom, which reflects the anisotropic resolution distribution along the azimuth angle. Furthermore, the checkers are large in both the longitudinal and latitudinal direction. The form of the convex mirror used in Kondo's anisotropic omnidirectional camera was determined with few coefficients. This demerit created nearly constant latitudinal resolution while longitudinal resolution changed significantly. Since our algorithm has many degrees of design freedom (the number of control variables), resolution distribution can be controlled more flexibly.

\section{Forming Non-smooth Surfaces}

Direct definition of the mirror surface with its gradients as control variables can represent a non-smooth surface and projection. Thus our method covers not 
168 Integrability-based Free-Form Mirror Design

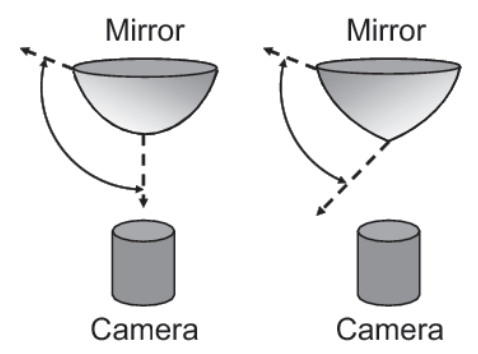

(a) two type omni-cameras

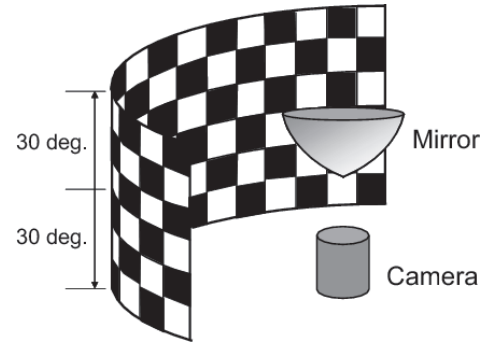

(b) setup for generating input images
Fig. 7 Omnidirectional camera with a spiny convex mirror. Correspondences between the input images and the scene is the same as Fig. 6.

only the single curved surface addressed in conventional methods such as ${ }^{7), 9), 10), 13)}$ but also a surface with sharp edges and/or vertexes. Three configurations are demonstrated to validate the performance of our algorithm with respect to nonsmooth surfaces.

\subsection{A Spiny Convex Mirror}

Generally, a conventional omnidirectional camera with a convex mirror covers an omnidirectional scene from directly under the camera to obliquely upward. While this FOV is appropriate to observe the whole scene including the ground, the camera itself is unfortunately also captured. Furthermore, observation of the ground is not necessary if we wish to capture only a horizontal scene. We can avoid capturing these unwanted scenes by forming a convex mirror that has a spiny bottom (Fig. 7 (a)). We have designed omnidirectional cameras with spiny convex mirrors designed using two separate algorithms: our method and Swaminathan's method ${ }^{13)}$. The desired projection uniformly covers panoramic scenes whose vertical FOV is \pm 30 degrees. Figure $\mathbf{8}$ (a) and (b) show a close-up of the bottom of the two convex mirrors. Our algorithm forms a sharper vertex. To confirm each vertical FOV, input images are generated by the setup shown in Fig. 7 (b). Take note of the center of the input image that corresponds to the downward scene. The black colored region at the center indicates superfluous FOV about the scene underneath. Since a smaller region appears in Fig. 8 (c) than in (d), our algorithm is better suited to forming spiny convex mirrors.

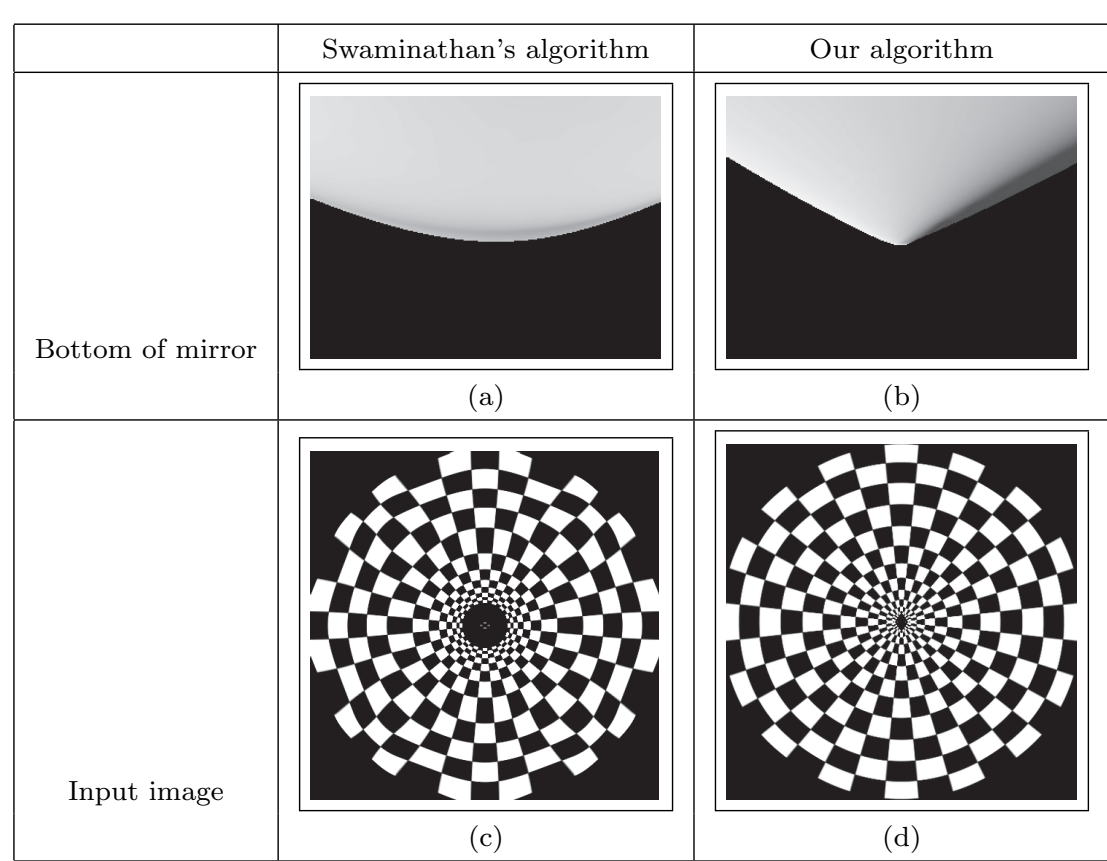

Fig. 8 Design results of the spiny convex mirrors.

\subsection{Pyramid Mirror}

We evaluate the design ability with respect to ridge lines by designing a composite mirror. A pyramid mirror is one of the simplest composite mirrors constructed from four flat mirrors, with four non-smooth ridge lines at their joints. Many residuals remain when redesigning the pyramid mirror shape from its own projection using the conventional method ${ }^{13)}$ that assumes a single curved mirror (Fig. $9(\mathrm{c})$ ). To avoid these residuals when using the conventional methods, each component mirror needs to be designed individually. This however, causes problems in the construction as continuity at the joints is not considered. On the other hand, our method suppresses residuals at the joints and allows them to expand to neighborhoods (Fig. 9 (d)) in spite of the single mirror design. The pyramid mirror, which is one of the simplest examples of composite mirrors, can be used to quantitatively evaluate residuals easily. 
169 Integrability-based Free-Form Mirror Design

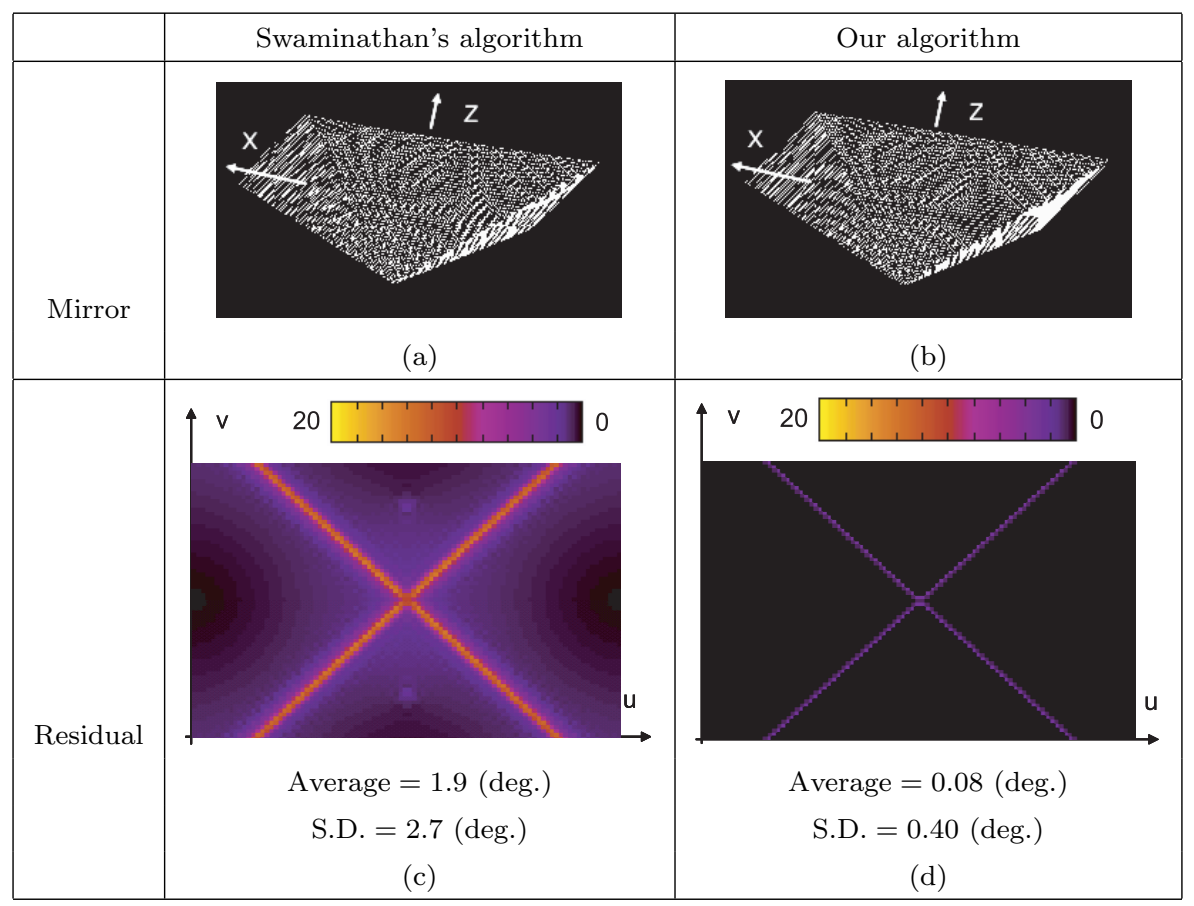

Fig. 9 Mirror design for discontinuous projection. (a), (c) Swaminathan's algorithm. Errors spread in bands around the boundaries. (b), (d) our algorithm. Error spreading is limited to narrow areas. The number of projection errors is small.

\subsection{Compound Eye Mirror}

The previous two configurations demonstrate the advantage of the proposed method with simple shape mirrors. Here we focus on designing an odd shaped mirror that has not been addressed in conventional methods. Assume that the image plane of a camera is divided into small rectangular semi-image planes and that each semi-image plane captures an environment with the same FOV. When the original image plane is divided into $4 \times 4=16$ semi-image planes, a $4 \times 4$ tiled image seems to appear in the image plane. We have attempted to construct a type of compound eye imaging system similar to an insect's eyes. We call this the "compound eye camera". The mirror is expected to have the

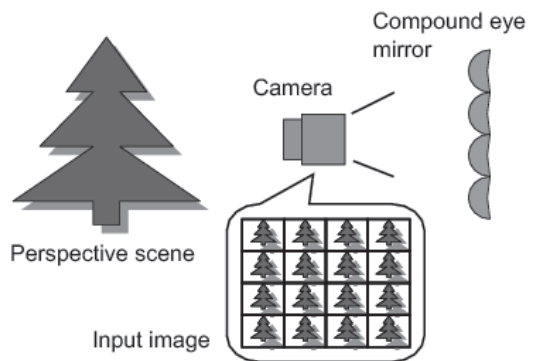

(a) compound eye configuration

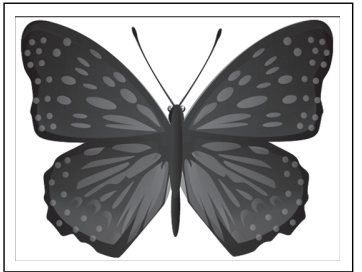

(c) target scene

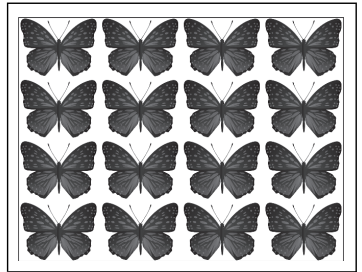

(d) ideal input image
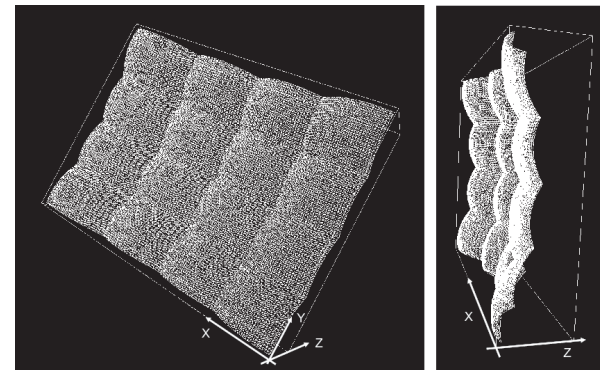

b) designed compound eye mirror
Fig. 10 Design of compound eye camera.

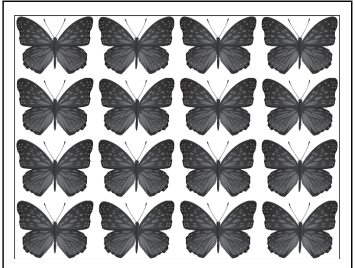

(e) input image with he designed mirror same number of convex shapes as there are divisions in the image plane. This configuration is illustrated in Fig. 10 (a). The convex shapes are not simple rotational symmetric shapes, but differ from one another, because each convex needs to produce the same FOV from different FOVs generated by each semiimage plane. This complicated and strange surface is difficult to create with conventional approaches using a single polynomial function ${ }^{7), 9), 10)}$ or the cross product of spline curves ${ }^{13)}$

We have designed a new type optical system with $4 \times 4$ semi-image planes as noted above. The desired FOV of horizontally \pm 30 degree $\times$ vertically \pm 23 degree for each semi-image plane results in a strangely shaped mirror that includes both smooth surfaces and sharp edges as shown in Fig. 10 (b). It consists of $4 \times 4$ different shaped convex surfaces as expected. The proposed method succeeded in limiting the average and variance of the projection residual to 1.0 degree and $3.0 \times$ $10^{-5}$ degree $^{2}$, respectively. Therefore the input image with the designed mirror 
(Fig. $10(\mathrm{e})$ ) is almost the same as that with the desired projection (Fig. $10(\mathrm{~d})$ ).

\section{Discussions}

\subsection{Differences from Conventional Methods for Projection Based} Mirror Design

We have already alluded to the differences between the proposed method and the conventional mirror design methods ${ }^{7), 9), 10), 13)}$. These are now discussed in detail.

There are, certainly, common aspects between the proposed method and the conventional ones, such as motivation, issues, and goals. However, the proposed method offers a significantly different mirror surface representation method Swaminathan's work ${ }^{13)}$ and the earlier works by Hicks ${ }^{7), 9)}$ define mirror surfaces using the cross product of spline curves and a single polynomial function or its multiplication based on the Reyleigh-Ritz approximation method. These definitions give an almost satisfactory approximation for a single smooth surface. But not all 3D surfaces can be covered with them. In fact, Hicks, et al. said that they had not considered an optimal choice of basis with the Rayleigh-Ritz approximation, and it seemed to be a difficult problem in their paper ${ }^{9)}$. Our method is one of a different approach to the surface representation problem. We directly define a mirror surface with a number of gradients, not with basis functions and their coefficients as used in the conventional methods. Although the conventional methods with polynomial functions and spline curves involve integrability conditions, forming a consistent surface is not guaranteed with only an assembly of gradients. Therefore we applied the additional constraints for integrability described in Eq. (10).

The proposed surface definition can easily represent non-smooth surfaces, including frequent bumps, sharp edges, and spiny vertexes (this has been demonstrated through the design examples discussed in Section 5). Non-smooth surfaces can be divided into a number of smooth surfaces, which are then dealt with individually. Unfortunately, this causes an additional problem, namely interrelations between the component mirrors, such as their positions and border connections. These interrelated problems are important issues when it comes to manufacturing a mirror and aligning it precisely. Whereas conventional methods do not even discuss these problems, the proposed method need not consider them as it is able to represent non-smooth surfaces. Even a complicated mirror constructed from a combination of component mirrors can still be designed as a single mirror. As clarified above, the surface representation approach underlying the proposed method is essentially different to the conventional method.

\subsection{Limitations and Future Works}

We have assumed that a mirror surface is defined as a single continuous surface with a set of gradients. Therefore, the proposed method can design a mirror with a large depth gap only if it is a single continuous surface. Multiple mirrors can, of course, be designed as a group of single mirrors or as a conjunction of the multiple mirror as if it were a single mirror, as described in the previous subsection. In this case, the proposed method works especially well when continuity in the borders of the component mirrors is a problem, such as the configuration given in Section 5 .

The proposed method cannot, however, design optics with multiple reflections by multiple mirrors, because of the assumption of only one reflection for each ray from the environment to an image plane as shown in Fig. 1. One suitable approach is to divide multiple reflections into a series of single reflections for sequential design.

We have designed various mirror shapes, but none of these have been manufactured as yet. We would like to evaluate the actual projections and captured images with real optical equipment. Designing and manufacturing mirrors for practical use is also envisaged.

\section{Conclusion}

In this paper, we proposed a design method for a free-form mirror surface for a catadioptric imaging system, using the assembly of its gradients. It is a new approach to defining 3D mirror surfaces for catadioptric camera design. We formulated the integrability constraint of the gradients to form a consistent surface in the mirror design, using a method similar to that used in gradient based shape reconstruction schemes. Our approach produces mirror surfaces with minimal overall projection residuals, and also works well for non-smooth mirrors such as spiny convex mirrors and compound eye mirrors.

Acknowledgments This work is supported by the Special Coordination 
Funds for Promoting Science and Technology of Ministry of Education, Culture, Sports, Science and Technology.

\section{References}

1) Yamazawa, K., Yagi, Y. and Yachida, M. HyperOmni Vision: Visual Navigation with an Omnidirectional Image Sensor, Systems and Computers in Japan, Vol.28, No.4, pp.36-47 (1997).

2) Nayar, S.K.: Catadioptric Omnidirectional Camera, Proc. IEEE Conf. on Computer Vision and Pattern Recognition, pp.482-488 (1997).

3) Iwata, H.: Full-Surround Image Display Technologies, International Journal of Computer Vision, Vol.58, No.3, pp.227-235 (2004).

4) Nakamura, T. and Ishiguro, H.: Automatic $2 \mathrm{D}$ map construction using a special catadioptric sensor, Proc. IEEE/RSJ Int. Conf. on Intelligent Robots and Systems, pp.196-201 (2002)

5) Gasper, J., Decco, C., Okamoto J. and Victor, J.S.: Constant Resolution Omnidirectional Cameras, Proc. IEEE Workshop on Omnidirectional Vision, pp.27-34 (2002).

6) Conroy, T.L. and Moore, J.B.: Resolution Invariant Surfaces for Panoramic Vision Systems, Proc. IEEE Int. Conf. on Computer Vision, Vol.1, pp.392-397 (1999).

7) Hicks, R.A., Perline, R.K. and Coletta, M.: Geometric Distributions for Catadioptric Sensor Design, Proc. IEEE Conf. on Computer Vision and Pattern Recognition, pp.584-589 (2001).

8) Hicks, R.A. and Perline, R.K.: Equi-areal Catadioptric sensors, Proc. IEEE Workshop on Omnidirectional Vision, pp.13-19 (2002).

9) Hicks, R.A. and Perline, R.K.: The method of vector fields for catadioptric sensor design with applications to panoramic imaging, Proc. IEEE Conf. on Computer Vision and Pattern Recognition, Vol.2, pp.143-150 (2004).

10) Hicks, R.A.: Designing a mirror to realize a given projection, Journal of Optical Society of America, Vol.22, No.2, pp.323-329 (2005).

11) Kondo, K., Yagi, Y. and Yachida, M.: Non-isotropic Omnidirectional Imaging System for an Autonomous Mobile Robot, Proc. IEEE Int. Conf. on Robot and Automation, pp.1240-1245 (2005).

12) Gaechter, S., Pajdla, T. and Micusik, B.: Mirror Design for an Omnidirectional camera with a space variant imager, Proc. IEEE Workshop on Omnidirectional Vision Applied to Robotic Orientation and Nondestructive Testing, pp.99-105 (2001).

13) Swaminathan, R., Nayar, S.K. and Grossberg, M.D.: Designing of Mirrors for catadioptric systems that minimize image error, Proc. IEEE Workshop on Omnidirectional Vision (2004).

14) Horovitz, I. and Kiryati, K.: Depth from gradient fields and control points: Bias correction in photometric stereo, Journal of Image and Vision Computing, Vol.22,
No.9, pp.681-694 (2004)

15) Agrawal, A. and Raskar, R.: An Algebraic Approach to Surface Reconstruction from Gradient Fields, Proc. IEEE Int. Conf. on Computer Vision, Vol.1, pp.174181 (2005).

16) Horn, B.K.P.: Robot Vision (MIT Electrical Engineering and Computer Science Series), MIT Pr.

17) Tankus, A., Sochen, N. and Yeshurun, Y.: A New Perspective [on] Shape-fromShading, Proc. IEEE Int. Conf. on Computer Vision, Vol.2, pp.862-869 (2003).

18) Tankus, A. and Kiryati, N.: Photometric Stereo under Perspective Projection, Proc. IEEE Int. Conf. on Computer Vision, Vol.1, pp.611-616 (2005).

\section{Appendix}

A general solution for a homogeneous linear equation can be calculated with the following procedures. Assume a homogeneous linear equation $\mathbf{A x}=\mathbf{0}$ with an $m \times n$ matrix $\mathbf{A}(m<n$, and $\operatorname{rank} \mathbf{A}=m)$. Since $\operatorname{rank} \mathbf{A}=\operatorname{row} \mathbf{A}=m, \mathbf{A}$ can be translated into the form given by

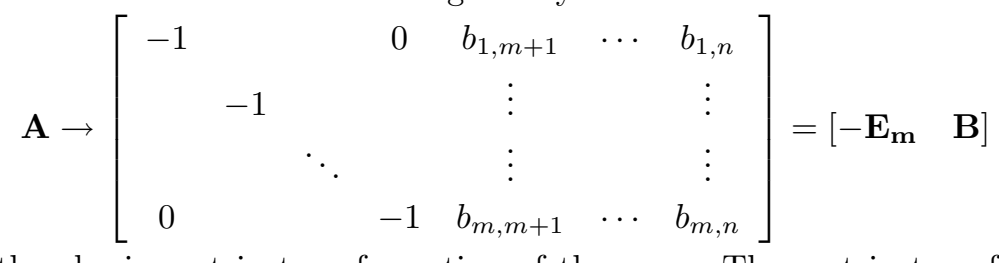

with a basic matrix transformation of the rows. The matrix transformation is a combination of three types of operations: swapping two rows, multiplying all elements of the target row by the same value, and adding one row to another. This transformation retains the properties of the original equation (i.e., $\mathbf{A x}=$ $\left.\mathbf{0} \Leftrightarrow\left[\begin{array}{ll}-\mathbf{E}_{\mathbf{m}} & \mathbf{B}\end{array}\right] \mathbf{x}=\mathbf{0}\right)$. If we assume the $n-m$ vectors to be 
172 Integrability-based Free-Form Mirror Design

$$
\mathbf{x}_{\mathbf{1}}=\left[\begin{array}{c}
b_{1, m+1} \\
\vdots \\
b_{m, m+1} \\
1 \\
0 \\
\vdots \\
\vdots \\
0
\end{array}\right], \mathbf{x}_{\mathbf{2}}=\left[\begin{array}{c}
b_{1, m+2} \\
\vdots \\
b_{m, m+2} \\
0 \\
1 \\
0 \\
\vdots \\
0
\end{array}\right], \cdots, \mathbf{x}_{\mathbf{n}-\mathbf{m}}=\left[\begin{array}{c}
b_{1, n} \\
\vdots \\
b_{m, n} \\
0 \\
\vdots \\
\vdots \\
0 \\
1
\end{array}\right]
$$

these are obviously linearly independent of each other and $\mathbf{A x}_{\mathbf{i}}=\mathbf{0}$ for $i=$ $1,2, \cdots, n-m\left(\operatorname{check}\left[-\mathbf{E}_{\mathbf{m}} \quad \mathbf{B}\right] \mathbf{x}_{\mathbf{i}}=0\right)$. Therefore $\mathbf{x}_{\mathbf{i}}, i=1,2, \cdots, n-m$ are vectors considered as elementary solutions or linearly independent solutions of $\mathbf{A x}=\mathbf{0}$ (linear algebra indicates that these are equal to bases in the solution space of $\mathbf{A x}=\mathbf{0})$. Finally a general solution $\mathbf{x}$ of $\mathbf{A x}=\mathbf{0}$ can be obtained as a linear combination of $\mathbf{x}_{\mathbf{i}}$. It is given by

$$
\mathbf{x}=\sum_{i=1}^{n-m} c_{i} \mathbf{x}_{\mathbf{i}}=\left[\begin{array}{llll}
\mathbf{x}_{\mathbf{1}} & \mathbf{x}_{\mathbf{0}} & \cdots & \mathbf{x}_{\mathbf{n}-\mathbf{m}}
\end{array}\right]\left[\begin{array}{c}
c_{1} \\
c_{2} \\
\vdots \\
c_{n-m}
\end{array}\right]=\left[\begin{array}{c}
\mathbf{B} \\
\mathbf{E}_{\mathbf{n}-\mathbf{m}}
\end{array}\right] \mathbf{y}=\mathbf{F} \mathbf{y}
$$

where $\mathbf{y}=\left[c_{1}, c_{2}, \cdots, c_{n-m}\right]^{T}$ is a coefficient vector that denotes the actual DOF of the solution for $\mathbf{A x}=\mathbf{0}$

(Received October 3, 2008)

(Accepted June 10, 2009)

(Released September 24, 2009)
Kazuaki Kondo received his M.E. and Ph.D. degrees from Osaka University in Japan. He became a research associate at Osaka University in 2007, and an assistant professor at Kyoto University in 2009. He was awarded the Kusumoto award in 2002. His research interests are computer vision and intelligent support on human communications. He is a member of IEICE.

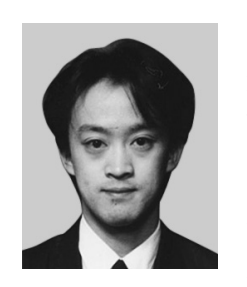

Yasuhiro Mukaigawa received his M.E. and Ph.D. degrees from University of Tsukuba in 1994 and 1997, respectively. $\mathrm{He}$ became a research associate at Okayama University in 1997, an assistant professor at University of Tsukuba in 2003, and an associate professor at Osaka University in 2004. His current research interests include photometric analysis and computational photography. He was awarded the MIRU Nagao Award in 2008. He is a member of IEICE, VRSJ, and IEEE.

(Communicated by Hiroshi Ishikawa) 
173 Integrability-based Free-Form Mirror Design

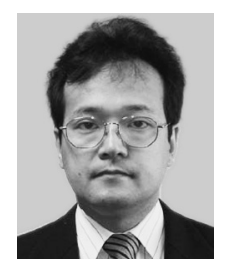

Yasushi Yagi is the Professor of Intelligent Media and Computer Science, and the Assistant Director of the Institute of Scientific and Industrial Research, Osaka University, Ibaraki, Japan. He received his Ph.D. degree from Osaka University in 1991. In

1985, he joined the Product Development Laboratory, Mitsubishi Electric Corporation, where he worked on robotics and inspections. He became a research associate in 1990, a lecturer in 1993, an associate professor in 1996, and a professor in 2003 at Osaka University. International conferences for which he has served as chair include: FG1998 (Financial Chair), OMINVIS2003 (Organizing chair), ROBIO2006 (Program co-chair), ACCV2007 (Program chair), PSVIT2009 (Financial chair), ICRA2009 (Technical Visit Chair) and ACCV2009 (General chair). He has also served as the Editor of IEEE ICRA Conference Editorial Board (2007, 2008, 2009). He is the Editor-in-Chief of IPSJ Transactions on Computer Vision \& Image Media and the Associate Editor-in-Chief of IPSJ Transactions on Computer Vision \& Applications. He was awarded ACM VRST2003 Honorable Mention Award, IEEE ROBIO2006 Finalist of T.J. Tan Best Paper in Robotics, IEEE ICRA2008 Finalist for Best Vision Paper, MIRU2008 Nagao Award. His research interests are computer vision, medical engineering and robotics. He is a member of IEICE, RSJ, and IEEE. 